ARTICULO DE INVESTIGACION

\title{
Análisis sobre el efecto del uso del suelo en la diversidad estructural del matorral espinoso tamaulipeco
}

\author{
Analysis of land use effect on the structural diversity \\ of Tamaulipan thornscrub
}

\author{
Javier Jiménez-Pérez1', Eduardo Alanís-Rodríguez², \\ Óscar Aguirre-Calderón1, Marisela Pando-Moreno1 \\ y Marco González-Tagle1
}

\begin{abstract}
RESUMEN
El presente estudio analiza el efecto del uso del suelo en la diversidad estructural del matorral espinoso tamaulipeco en el noreste de México. El objetivo fue determinar la modificación de la diversidad, indicadores ecológicos, estructura dimensional y la distribución vertical de las especies arbóreas y arbustivas debido al cambio de uso del suelo. El análisis se realizó mediante la comparación de tres áreas con distinto historial de uso: ganadería extensiva, agricultura tradicional y matarrasa, con un abandono de 21 años. La metodología propuesta fue la combinación de sitios rectangulares y el análisis muestral denominado "grupo estructural de los cuatro árboles". El análisis estructural comparativo mostró evidencias significativas que indican que el cambio de uso del suelo trae como consecuencia que las áreas presenten diferente diversidad, indicadores ecológicos, distribución vertical y estructura dimensional.
\end{abstract}

PALABRAS CLAVE:

Diferenciación dimensional, distribución vertical de especies, diversidad de especies, indicadores ecológicos.

\begin{abstract}
The effect of land use on the structural diversity of Tamaulipan thornscrub in northeastern Mexico was examined. The objective was to determine whether land use modified diversity, ecological indicators, structural and vertical distribution of trees and shrubs. The analysis was performed by comparing three ecosystems with different land use history: extensive cattle grazing, traditional agriculture and clear cutting with 21 year of abandonment. The methodology used was a combination of rectangular sites and a sampling analysis called "structural group of four trees". Results showed differences in diversity, ecological indicators, vertical distribution and dimensional structure.
\end{abstract}

KEY WORDS:

Dimensional difference, vertical species distribution, species diversity and ecological indicators. 


\section{INTRODUCCIÓN}

El matorral espinoso tamaulipeco (MET) de la planicie semiárida de la región Noreste de México está dominado por una diversidad bastante densa, de 15000 a 21000 individuos/ha de especies arbóreas-arbustivas, 33 especies y subarbustivas, con 31 especies (Heiseke y Foroughbakhch, 1985; González et al., 1997; González y Cantú, 2001; Alanís, 2006). Este tipo de ecosistema cubre una superficie de $200000 \mathrm{~km}^{2}$ del noreste de México y sur de Texas, desde Llera de Canales y los límites de la Sierra Azul en Tamaulipas (González, 1985), hasta el Altiplano Edwards (Edwards Plateau) en Texas (Diamond et al., 1987), y de la Sierra Madre Oriental hasta el Golfo de México (Jurado y Reid, 1989). El MET tiene un uso tradicional silvoagropecuario, desde fines del siglo XVI, su importancia en la economía del noreste de México ha dado lugar a que haya sido fragmentado por décadas para dar al suelo uso agrícola y ganadero y las superficies remanentes rara vez son de vegetación primaria, ya que han recibido un impacto histórico de sobrepastoreo, agricultura, incendios forestales, extracción selectiva de componentes leñosos y forrajeros o por la eliminación a matarrasa del matorral (Rzedowski, 1981; Foroughbakhch y Peñaloza, 1988; García y Jurado, 2008; Alanís et al., 2008a).

Una manera de estimar la condición de los ecosistemas en un momento determinado y su evolución en el tiempo ha sido mediante la caracterización de la estructura de los ecosistemas (Gadow et al., 1998). Para ello se consideran índices estructurales y variables dasométricas, que incluyen diámetro, altura, área basal, densidad, etc., a fin de lograr una mejor descripción (Aguirre et al., 2003a). La caracterización de la estructura de ecosistemas forestales constituye una condición inicial para la toma de deci- siones sobre el manejo sustentable de los recursos naturales (Aguirre et al., 2003b). Los métodos de caracterización pueden ser distintos en función de los objetivos (Del Río et al., 2003), pudiendo incluir índices de diversidad, mezcla de especies, indicadores ecológicos, perfil de especies y diferenciación dimensional, que pueden reproducir con diferente precisión la condición del ecosistema objeto de estudio (Kramer et al., 1999; Jiménez et al., 2001a; Solís et al., 2006).

A nivel mundial existe suficiente literatura sobre la estructura de ecosistemas forestales, como la de Huang et al. (2003), Grela (2003), Graz (2004), Montes et al. (2004), Mani y Parthasarathy (2006), Vorcák et al. (2006) y Mason et al. (2007), que en su mayoría se encuentran enfocados a ecosistemas tropicales. En México, los ecosistemas más estudiados también son los de tipo tropical. Un ejemplo son los estudios de Segura et al. (2003), Durán et al. (2006), Van et al. (2006) y Castellanos et al. (2008), seguidos de los templados como los de Aguirre et al. (1998), Jiménez et al. (1998), Jiménez et al. (2001b), Aguirre et al. (2003a), Aguirre et al. (2003b), Corral et al. (2005) y González et al. (2006). Sobre el MET existen escasos estudios que evalúen la estructura, como el de Alanís et al. (2008a), donde se evaluaron índices de riqueza y diversidad del estrato arbóreo. De ahí que el presente estudio tenga como objetivos los que a continuación se mencionan.

\section{OBJETIVOS}

El presente estudio tiene dos objetivos: (1) describir la diversidad estructural de tres áreas con distinto historial de uso en el matorral espinoso tamaulipeco y (2) realizar un análisis que permita conocer el efecto del uso del suelo sobre la riqueza de especies, diversidad biológica e indicadores ecológicos. 


\section{METODOLOGÍA}

El trabajo de investigación se desarrolló en el municipio de Linares, N. L., entre las coordenadas $25^{\circ} 09^{\prime}$ y $24^{\circ} 33^{\prime}$ de latitud norte, y $99^{\circ} 54^{\prime}$ y $99^{\circ} 07^{\prime}$ de longitud oeste, con una altitud de $350 \mathrm{msnm}$. Las especies que destacan por su abundancia y cobertura son: Acacia rigidula, Acacia farnesiana, Pithecellobium pallens, Cordia boissieri, Karwinskia humboldtiana y Prosopis laevigata (González et al., 1997; Espinoza y Navar, 2005; Alanís et al., 2008a).

La evaluación del MET se realizó en el año 2005 en tres áreas con distinto historial de uso antropogénico que tenían un abandono de 21 años, los cuales fueron: 1) ganadería extensiva, 2) agricultura y 3) matarrasa. El área de ganadería extensiva estuvo influenciada por la extracción selectiva de sus componentes leñosos y la acción del ramoneo y pisoteo de ganado caprino. El área de agricultura se desmontó con maquinaria agrícola, removiendo el suelo y cultivando maíz y sorgo de temporada durante un periodo de cinco años. El área de matarrasa se desmontó con maquinaria agrícola y no presentó ninguna actividad silvoagropecuaria. Las áreas de agricultura y matarrasa tenían una distancia aproximada de 200 metros de las áreas fuentes semilleras, mientras que el área de ganadería estaba rodeada de material germoplásmico. Las fuentes semilleras son zonas del MET con vegetación secundaria. Las tres áreas se presentaban entre los 350 msnm y los $380 \mathrm{msnm}$, con pendiente < $3 \%$ y suelo vertisol.

La metodología para realizar la presente investigación fue una combinación de sitios rectangulares de $10 \mathrm{~m} \times 25$ $\mathrm{m}$, y el análisis muestral propuesto por Köhler en 1951 y desarrollado por Füldner y Gadow (1994). La forma rectangular de los sitios se utilizó debido a su facilidad de delimitación y medición en vegetación densa, con respecto a la forma circular (Alanís et al., 2008b). Para que las variables topográficas, edáficas y altitudinales fueran homogéneas en los sitios de muestreo, la distribución fue dirigida, teniendo una distancia aproximada de $50 \mathrm{~m}$ entre ellos. Para la obtención del número mínimo de sitios de muestreo se elaboró una curva especies-área para cada una de las tres áreas consideradas en el estudio, siguiendo el criterio de Mueller y Ellenberg (1974). Se realizaron cuatro sitios de muestreo por área, sumando un total de 12 sitios de muestreo para el estudio.

En las mismas áreas donde se realizó el muestreo por parcelas se efectuó el muestreo denominado "grupo estructural de los cuatro árboles" para la estimación de los parámetros estructurales, el cual consistió en realizar una red de proyecciones de coordenadas en la abscisa y ordenada con una equidistancia de $10 \mathrm{~m}$, donde sus puntos de intersección fueron la base para la ubicación del centro de la unidad muestral. Al árbol más próximo a los puntos de intersección de la red se le denominó árbol-muestra y partiendo de éste se determinó la distancia de los tres individuos más cercanos, conformando el grupo estructural de los cuatro árboles (Füldner y Gadow, 1994). El primer punto de muestreo se estableció en la esquina noreste del primer sitio rectangular de cada área. Para la obtención del número mínimo de unidades muestrales se elaboró una curva especies-área para cada una de las tres áreas consideradas en el estudio (Mueller y Ellenberg, 1974), resultando 39 unidades muestrales por comunidad, sumando en total 117 unidades en la investigación.

El muestreo por sitios rectangulares se empleó para calcular el índice de diversidad de Shannon $\left(H^{\prime}\right)$ y el índice de 
distribución vertical de especies $(A)$, mientras el muestreo "grupo estructural de los cuatro árboles" se utilizó para estimar los índices de mezcla de Gadow $\left(M_{i}\right)$, índices de diferenciación diamétrica $\left(T D_{i}\right)$ y en altura $\left(T H_{i}\right)$ y los indicadores ecológicos de abundancia $\left(A R_{i}\right)$, dominancia $\left(D R_{i}\right)$, frecuencia $\left(F R_{i}\right)$ e índice de valor de importancia (IVI).

Se efectuaron mediciones dasométricas de altura total $(h)$ y diámetro $\left(d_{0}, 10\right)$. La medición del diámetro se efectuó a $0,10 \mathrm{~m}$ sobre la base del suelo, siendo una medida estándar empleada para especies arbóreas y arbustivas del MET (Gómez, 2000; Ruiz, 2005; Alanís et al., 2008a) y se evaluaron los individuos con un diámetro $\mathrm{d}_{0,10} \geq 1 \mathrm{~cm}$, para obtener una mayor representatividad de las especies. A continuación se presentan los índices de diversidad estructural y los indicadores ecológicos correspondientes.

\section{Diversidad de especies}

Índice de Shannon $\left(\mathrm{H}^{\prime}\right)$

Para estimar la diversidad de especies se utilizó el índice de Shannon \& Weiner (1948) mediante la ecuación:

$$
H^{\prime}=-\sum_{i=1}^{S} p_{i}^{*} \ln \left(p_{i}\right)
$$

Donde $S$ es el número de especies presentes, In es logaritmo natural y pi es la proporción de las especies $p_{i}=n_{i} / N$; donde $n_{i}$ es el número de individuos de la especie $i$ y $N$ es el número total de individuos.

El parámetro de abundancia de las especies de las diferentes comunidades se utilizó para comparar las áreas de estudio mediante un análisis de varianza
ANVA $(p \geq 0,05)$. Los datos se sometieron a una transformación logarítmica $\left(y^{\prime}=\right.$ $\left.\log \left(x_{i}+1\right)\right)$, para lograr que los datos cumplieran con las características de normalidad (Kolmogorv-Smirnof; $p=0,05$ ) aditividad y homocedasticidad (Zar, 1999). Cuando se determinaron diferencias significativas en el análisis de varianza se efectuaron comparaciones múltiples aplicando una Prueba de Tukey (Zar, 1999).

Índice de mezcla de Gadow $\left(M_{i}\right)$

Este indicador ecológico fue desarrollado por Gadow (1993) y Füldner (1995) para el muestreo estructural, considerando los tres vecinos del árbolmuestra, donde se evalúa la diversidad de especies de la vecindad de un árbol de referencia, y se define como la proporción de los tres vecinos que no pertenecen a la misma especie del árbol de referencia. Esta comparación corresponde a 0 si los vecinos pertenecen a la misma especie, 0,33 si un individuo pertenece a otra especie, 0,67 si dos de los tres vecinos pertenecen a otra especie y 1 cuando todos los individuos pertenecen a distintas especies. Para la obtención del promedio del índice porcentual de mezcla de especies se utilizó la ecuación matemática:

$$
M_{i}=\frac{1}{n} \sum_{j=1}^{n} V_{i j}
$$

Donde:

$$
V_{i j}=\left\{\begin{array}{l}
O, j^{\prime} \text { avo vecino pertenece a la misma especie } \\
1, j^{\prime} \text { avo vecino pertenece a otra especie }
\end{array}\right.
$$

\section{Estructura dimensional (TD y TH)}

La diferenciación dimensional permite describir la heterogeneidad dentro de la estructura del ecosistema, teniendo como 
base las relaciones dasométricas de las especies próximas (Pommerening, 1997; Jiménez et al., 2001b; Corral et al., 2005). Con fundamento en lo señalado anteriormente, la diferenciación diamétrica y de altura se definen como el resultado de la relación existente entre las variables dendrométricas $\left(d_{0,10}, h\right)$ del árbol-muestra y el primer vecino.

$$
\begin{gathered}
T D_{(i)}=\frac{1}{N} \sum_{N} \frac{1}{n} \sum_{n}\left(1-\frac{d \min _{\left(d_{0.10}\right)}}{d \max _{\left(d_{0.10}\right)}}\right) \\
T H_{(i)}=\sum_{N} \frac{1}{n} \sum_{n}\left(1-\frac{h \min }{h \max }\right)
\end{gathered}
$$

donde $T D_{(i)}$ y $T H_{(i)}=$ diferenciación diamétrica y en altura del área $i$; $n=$ número de vecinos considerados; $N=$ número de árboles medidos; $d$ min, $d$ max = diámetro menor y mayor entre $i \mathrm{y}$ cada uno de sus $n$ vecinos; $h$ min, $h$ max $=$ altura menor y mayor entre $i$ y cada uno de sus $n$ vecinos.

Los valores de la diferenciación diamétrica y de altura se incrementan al aumentar la diferencia media de los tamaños de los árboles cercanos al árbol de referencia. Un valor de cero corresponde a una situación donde los árboles presentan la misma dimensión. Se integraron cinco categorías de diferenciación dimensional de acuerdo con Aguirre et al. (1998) y Jiménez et al. (1998): (Escasa: 0,0 < TDi y THi < 0,2; Moderada: 0,2 < TDi y THi < 0,4; Media: 0,4 $<$ TDi y THi < 0,6; Alta 0,6 < TDi y THi < 0,8; Muy alta 0,8 $<$ TDi y $\mathrm{THi}<1,0$ ).

Índice de distribución vertical de especies (A)

Para la caracterización de la estructura vertical de las especies se utilizó el índice de distribución vertical de especies (A) (Pretzsch, 1996; Del Río et al., 2003).

$$
A=-\sum_{i=1}^{S} \sum_{j=1}^{Z} p_{i j} * \ln \left(p_{i j}\right)
$$

donde $S=$ número de especies presentes; $Z$ = número de estratos de altura; $p_{i j}=$ porcentaje de especies en cada zona, y se estima mediante la siguiente ecuación $p_{i j}=n_{i, j} / N$; donde $n_{i, j}=$ número de individuos de la misma especie $(i)$ en la zona $(j)$ y $N=$ número total de individuos. Para la estimación de la distribución vertical de las especies se definieron tres zonas de altura (Pretzsch, 1996; Jiménez, 2001b), siendo éstas: zona I: $80 \%-100 \%$ de la altura máxima del área; zona II: $50 \%-80 \%$, y zona III: 0 a $50 \%$. A toma valores entre 0 y un valor máximo $\left(A_{\max }=\ln \left(S^{\star} Z\right)\right)$. Un valor $A=0$ significa que el rodal está constituido por una sola especie que ocurre en un solo estrato. $A_{\max }$ se alcanza cuando la totalidad de las especies ocurren en la misma proporción tanto en el rodal como en los diferentes estratos (Corral et al., 2005).

\section{Indicadores ecológicos}

Para evaluar el significado de las especies en la población se utilizó la estimación de los indicadores ecológicos: abundancia, dominancia, frecuencia e índice de valor de importancia como medida de valoración (Mueller y Ellenberg, 1974; Jiménez et al., 2001b; Magurran, 2004). Para la estimación de la abundancia relativa se empleó la siguiente ecuación matemática:

$$
\begin{gathered}
A_{i}=N_{i} / S \\
A R_{i}=\left(A_{i} / \sum A_{i}\right) * 100 \\
\mathrm{i}=1 \ldots \mathrm{n}
\end{gathered}
$$


Donde $A R_{i}$ es la abundancia relativa de la especie $i$ respecto a la abundancia total, $N_{i}$ es el número de individuos de la especie $i, S$ la superficie (ha). Para estimar la dominancia relativa $\left(D R_{i}\right)$ se emplea (Edwards et al., 1993):

$$
\begin{gathered}
D_{i}=A b_{i} / S\left(m^{2} / h a\right) \\
D R_{i}=\left(D_{i} / \sum D_{i}\right) * 100 \\
i=1 \ldots . \mathrm{n}
\end{gathered}
$$

donde $D R_{i}$ es la dominancia relativa de la especie $i$ respecto a la dominancia total, $A b$ el área basal de especie $i$ y $S$ la superficie. La frecuencia relativa $\left(F R_{i}\right)$ se obtiene con la siguiente ecuación (Franco et al., 1989):

$$
\begin{gathered}
F_{i}=P_{i} / N S \\
F R_{i}=\left(F_{i} / F_{i}\right) * 100 \\
\mathrm{i}=1 \ldots . \mathrm{n}
\end{gathered}
$$

donde $F R_{i}$ es la frecuencia relativa de la especie $i$ respecto a la frecuencia total, $P_{i}$ la frecuencia de la especie $i$ en los sitios de muestreo y NS el número total de sitios de muestreo. El índice de valor de importancia (IVI) se define como (Franco et al., 1989):

$$
I V I=A R_{i}+D R_{i}+F R_{i}
$$

donde $A R_{i}$ es la abundancia relativa, $D R_{i}$ es la dominancia relativa y $F R_{i}$ la frecuencia relativa.

\section{RESULTADOS Y DISCUSIÓN}

En total se registraron 17 familias, 23 géneros y 27 especies, la familia más representativa fue Leguminosae con 5 especies, la alta presencia de esta familia en áreas con vegetación secundaria relativamente joven puede relacionarse con diversos factores como escasa disponibilidad de nutrientes en el suelo, intolerancia a la sombra y mecanismos relacionados con la reproducción (Alanís, 2006). Estos resultados concuerdan con los de González et al. (1997) y García y Jurado (2008), quienes mencionan que las áreas que han sido despojadas de su cobertura vegetal natural y posteriormente sometidas a uso agrícola y pecuario al ser abandonadas es probable que presenten una baja disponibilidad de nitrógeno; por lo tanto especies capaces de fijar nitrógeno atmosférico (característica común en las leguminosas), frecuentemente están presentes en las primeras fases de la sucesión.

De las 27 especies arbóreas y arbustivas, 24 se presentaron en el área de ganadería, 20 en matarrasa y 19 en agricultura, compartiendo 14 especies. El área con mayor densidad (16,983 ind/ha), área basal $\left(29,80 \mathrm{~m}^{2} / \mathrm{ha}\right)$, altura $(2,6 \mathrm{~m} \pm 0,64$ $\mathrm{m})$ y diámetro $(4,44 \mathrm{~m} \pm 1,72 \mathrm{~cm})$ fue ganadería, debido probablemente a que fue la única área que no se desmontó en su totalidad y tiene una fuente semillera adyacente, por lo tanto se encuentra en una etapa de recuperación más dinámica, donde hay una mayor competencia por los factores ambientales. Bernardia myricaefolia presenta la mayor densidad de individuos $(7,346 \mathrm{ind} / \mathrm{ha})$, ya que es una especie frecuentemente consumida y dispersada por el ganado (Ramírez et al., 2006) y al tener condiciones favorables para su germinación presentó alta abundancia.

El área con historial de agricultura presentó 2370 ind/ha y $156 \mathrm{~m} 2 / \mathrm{ha}$, 
siendo Diospyros texana la especie más abundante (660 ind/ha). El área matarrasa presentó 6230 ind/ha y 17 m²/ha, siendo Mimosa monancistra la especie con mayor presencia, lo cual concuerda con Camargo y García (2001) y Flores et al. (2006), quienes mencionan que es una especie oportunista y típicamente secundaria que coloniza áreas abiertas resultantes de diversas actividades antrópicas, debido principalmente a que la semilla germina en pocos días y el crecimiento es rápido, además tienen el potencial de regenerarse vegetativamente a partir de tocones y raíces.

\section{Diversidad de especies}

\section{A) Índice de Shannon $\left(H^{\prime}\right)$}

Los valores del índice de Shannon fueron 2,10 para ganadería extensiva, 2,24 para agricultura y 2,27 para matarrasa. El parámetro de abundancia de las especies de las comunidades se utilizó para comparar las áreas de estudio mediante el análisis de varianza ANVA. Los resultados mostraron que existen diferencias significativas $(P=0,019)$ entre las áreas de estudio, por lo que se aplicó la prueba de Tukey $(p \leq 0,05)$ para determinar las áreas con diferencias estadísticas, mostrando que el área de mata- rrasa no mostró diferencias significativas con agricultura y ganadería, mientras que agricultura y ganadería sí presentaron diferencia. Una explicación probable es que el área con historial de matarrasa únicamente fue desmontada y no hubo ningún impacto silvoagropecuario, mientras agricultura y ganadería presentaron diferentes impactos debido a su utilización productiva, lo que conllevó a estas diferencias en su diversidad.

\section{B) Índice de mezcla de Gadow $\left(M_{i}\right)$}

Este índice varía de 0 a 1 , cuanto más próximo a 1 mayor es la mezcla de especies y cuanto más próximo a 0 más se agrupan las especies (Del Río et al., 2003). En la figura 1 se presenta la distribución de los índices de mezcla individuales para las tres áreas. Las áreas muestran la misma tendencia de vecindad, ya que $67,9 \%$ de los individuos de las comunidades presentan 2 o 3 vecinos diferentes a él, mientras que menos de $5,1 \%$ se distribuye con tres vecinos de la misma especie. Los valores promedio fueron $M i_{G}=0,62, M i_{M}=0,63 \mathrm{y}$ $M i_{A}=0,64$ con lo que se puede aludir que las tres áreas muestran especies con una alta competencia interespecífica, ya que presentan vecindad con especies diferentes a la suya.

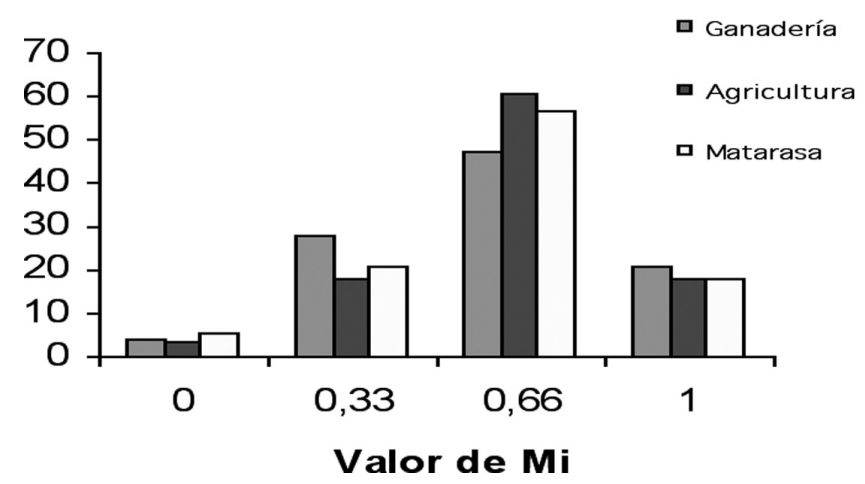

Figura 1. Distribución de la frecuencia porcentual de los valores del índice de mezcla de Gadow en las áreas evaluadas. 


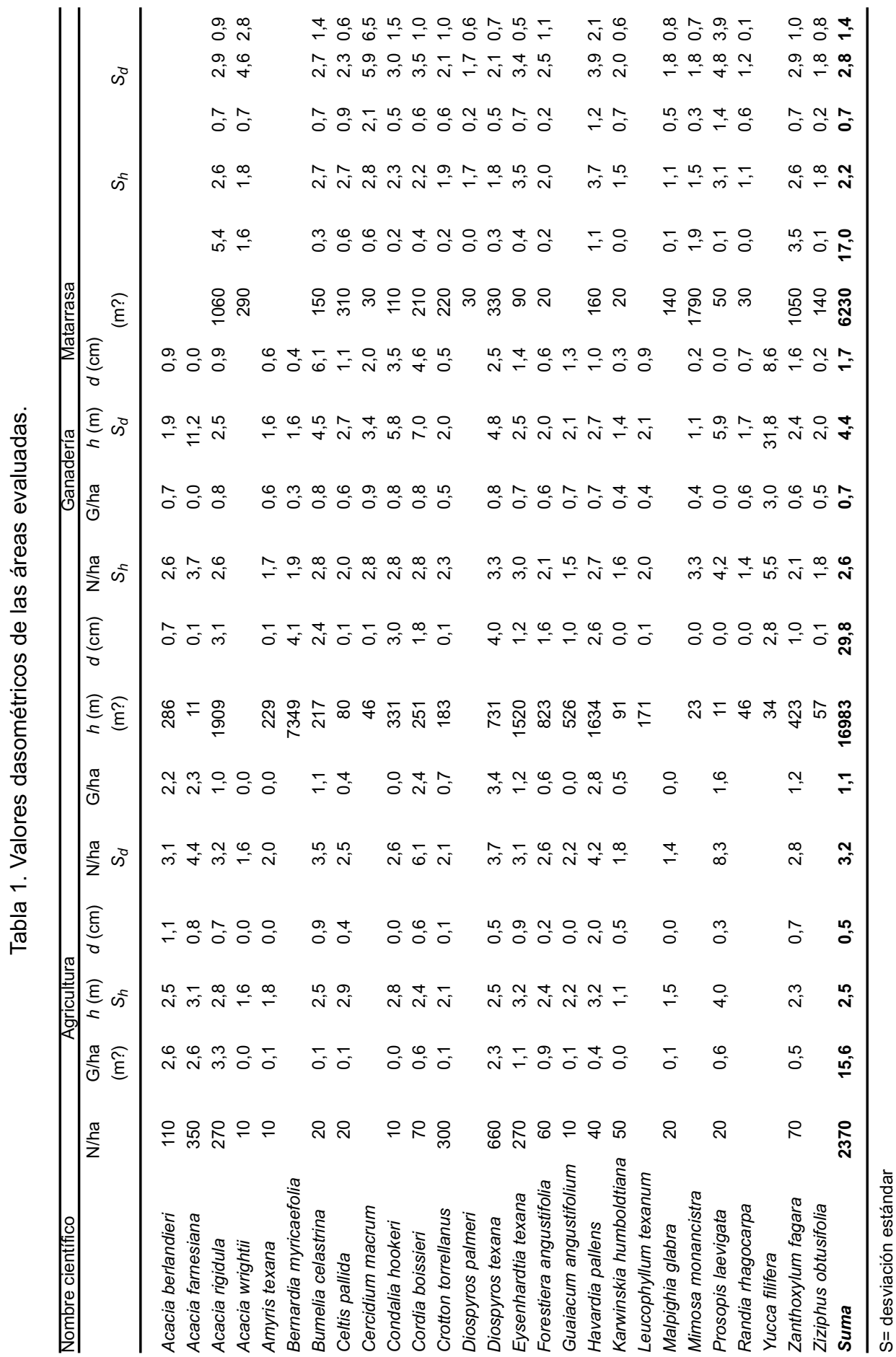




\section{Indicadores ecológicos}

La abundancia (n/ha), dominancia $\left(\mathrm{m}^{2} / \mathrm{ha}\right)$, frecuencia $\left(\mathrm{N}_{\mathrm{s}}\right)$ e índice de valor de importancia $(I V I)$ de las especies se utilizó para el análisis de las relaciones existentes entre las especies de las tres áreas evaluadas. El área con historial de ganadería extensiva (Tabla 2) presentó 24 especies, siendo las de mayor peso ecológico Bernardia myricaefolia $(79,77 \%)$, Eysenhardtia texana $(34,44 \%)$, Acacia rigidula $(34,25 \%)$ y Havardia pallens $(25,16 \%)$, sumando $58 \%$ del total del valor de importancia. Esta área al no haber sido desprovista de su vegetación, se encuentra en una etapa más dinámica, donde los individuos están en mayor competencia por espacio.

En el área con historial de agricultura (Tabla 2) se presentaron 16 especies. Las de mayor valor de importancia son Diospyros texana $(64,57 \%)$, Acacia farnesiana $(49,03 \%)$, Acacia rigidula $(48,07 \%)$ y Acacia berlandieri $(29,28 \%)$. Tres de las cuatro especies con mayor valor de importancia son de la familia Leguminosae, las cuales se distinguen por estar en las primeras etapas sucesionales y tener la capacidad de desarrollarse en áreas desprovistas de cobertura vegetal y suelos con bajo contenido de nitrógeno. Esto coincide con González et al. (1997) y Estrada et al. (2004) quienes mencionan que las especies con mayor densidad, cobertura y frecuencia de la vegetación con historial de agricultura en el MET son Acacia farnesiana y A. rigidula.

El área con historial de matarrasa presentó 20 especies (Tabla 2). Las de mayor importancia son Acacia rigidula (66,52\%), Mimosa monancistra (58,51\%), Zanthoxylum fagara $(47,95 \%)$ y Acacia farnesiana $(21,21 \%)$. En esta área, dos de las cuatro especies con mayor importancia pertenecen a la familia Legumi- nosae, ya que como se menciona anteriormente, tiene la capacidad de establecerse en espacios sin cobertura vegetal.

Los resultados de esta investigación indican que las actividades silvoagropecuarias modifican la presencia y peso ecológico de las especies, ya que existen especies como Bernardia myricaefolia que es muy abundante $(43,01 \%)$ y frecuente $(23,10 \%)$ en el área de ganadería, pero no tiene presencia en agricultura y matarrasa. Los resultados de la presente investigación comparados con un ecosistema de MET prístino como el evaluado por García y Jurado (2008) muestran diferencias en la presencia de especies, ya que las cuatro especies más abundantes de un ecosistema prístino son Helietta parvifolia (24,98\%), Gochnatia hypoleuca (14,93\%), Acacia rigidula (11,3\%) y Fraxinus greggii (10,18\%), de las cuales sólo Acacia rigidula se encuentra tanto en el área prístina e impactada, las tres especies restantes no tienen presencia en áreas impactadas.

\section{Estructura dimensional (TD y $T H$ )}

La figura 2 muestra la distribución de los valores del índice de diferenciación en diámetro de las tres áreas evaluadas, donde se observa que los valores mayores se encuentran en una diferenciación dimensional escasa y decrecen conforme se incrementa el valor de $T D_{i}$; lo que indica que la mayoría de los individuos presenta uniformidad de dimensión. Los promedios de diferenciación fueron de $T D_{A}=0,26, T D_{G}=0,26$ y $T D_{M}=0,33$. Una explicación de la diferenciación diamétrica escasa en el área de agricultura y matarrasa es que las áreas fueron desprovistas de su vegetación original y los individuos tuvieron las mismas condiciones ambientales para su desarrollo, presentando así una alta similitud en diámetros y posiblemente en edad. E 


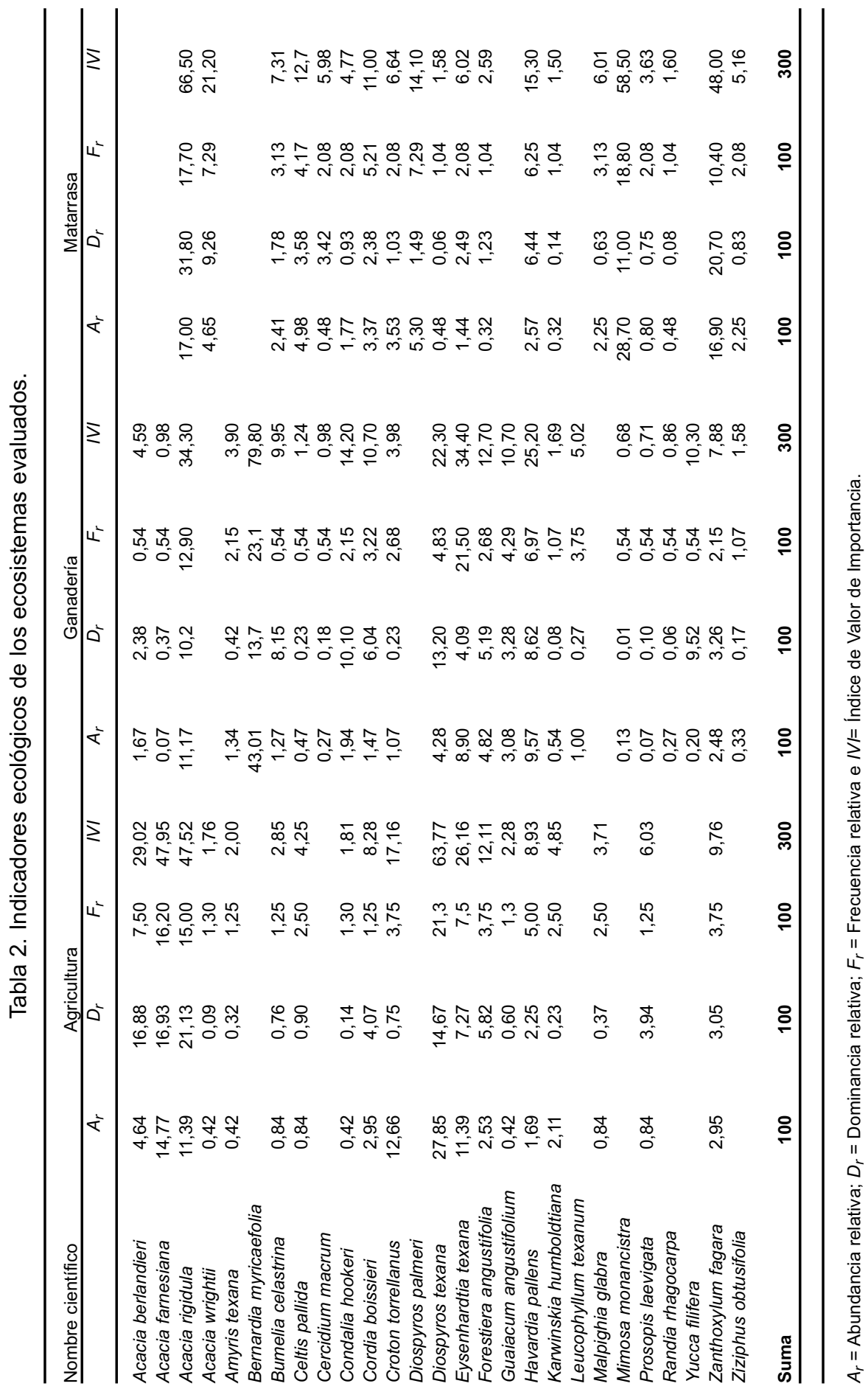


área de ganadería fue la única que no se desmontó, por lo tanto, después del cese de la actividad pecuaria hubo un alto establecimiento de individuos, los cuales muestran alta similitud en diámetros.

En el índice de diferenciación en altura $\left(T H_{i}\right)$ se presenta la misma tendencia que en la diferenciación diamétrica, ya que los valores más altos se ubican en una diferenciación dimensional escasa y decrecen conforme aumenta el valor de $\mathrm{TH}_{i}$ (Figura 3). Los promedios de diferenciación fueron de $T H_{A}=0,19$, $T H_{G}=0,23$ y $T H_{M}=0,27$. Esto corrobora el resultado del índice de distribución vertical de especies $(A)$, que indica que más del $58 \%$ de los individuos se localizan en un solo estrato, mostrando una fuerte competencia por la captación lumínica, formando una comunidad vegetal con una cohorte dominante.

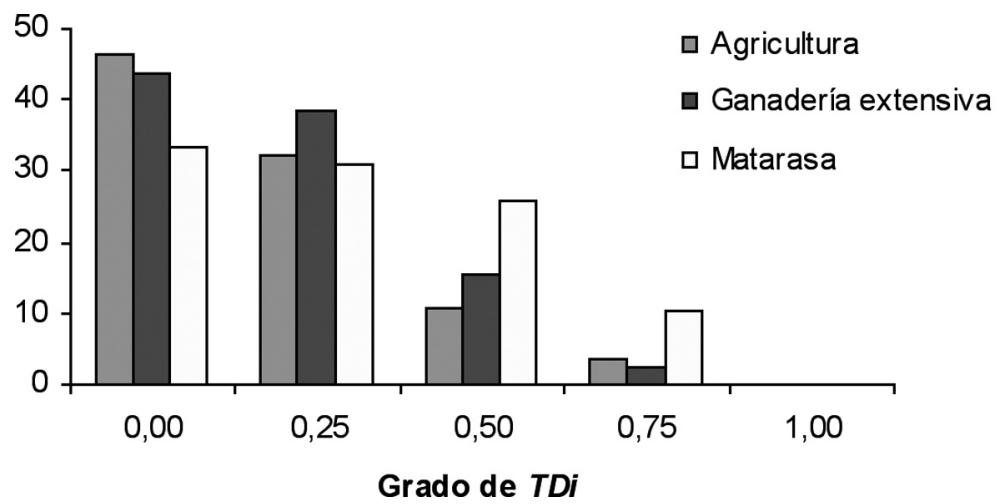

Figura 2. Distribución de la frecuencia porcentual de los valores del grado de diferenciación en diámetro TDi de las áreas evaluadas.

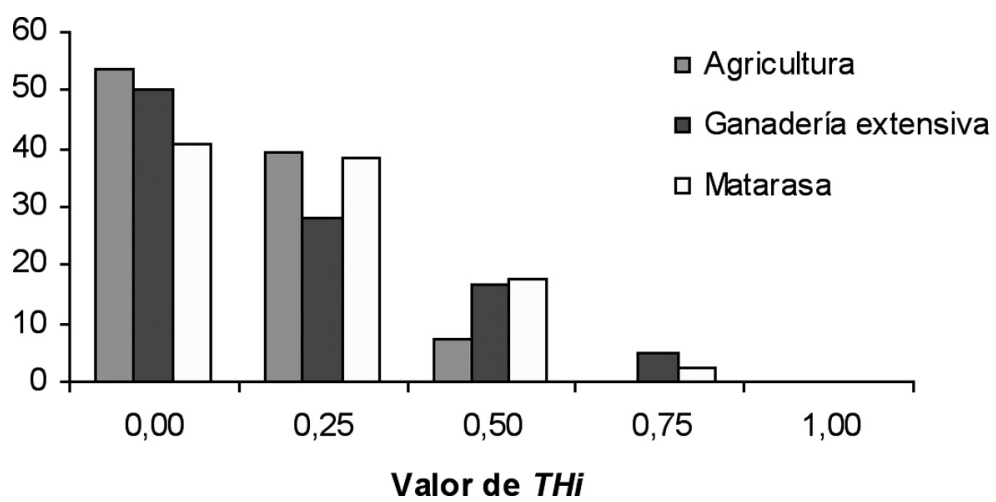

Figura 3. Distribución de la frecuencia porcentual de los valores del grado de diferenciación en altura $T H_{i}$ de las áreas evaluadas. 


\section{Índice de distribución vertical de especies $(A)$}

Para determinar si las áreas evaluadas son multicohortales fue necesario realizar un análisis de los estratos (Corral et al., 2005). En el análisis se distribuyen las especies en tres estratos. En el primero, $80 \%-100 \%$ de la altura máxima del área. En el segundo, 50\%-80\%, y en el tercero, de $0 \%$ a $50 \%$. En la figura 4 se presenta la abundancia porcentual de los individuos por estrato de cada área evaluada, donde se muestra que únicamente se presentan dos estratos (II y III) en las áreas con historial de agricultura y matarrasa y uno (III) en ganadería extensiva. En el estrato I del área con historial de agricultura se ubica $2,11 \%$ de los individuos, en ganadería extensiva un $0,13 \%$ y en matarrasa $6,24 \%$. El valor de A para agricultura es de 2,82 y de 2,85 para matarrasa, con valores de $A_{\max }$ de 4,04 y 4,09 respectivamente; lo que indica que estas áreas muestran dos estratos verticales. En el caso de ganadería extensiva se tiene un valor de $A$ menor $(2,25)$ y de $A_{\max }$ mayor $(4,27)$ en comparación con las otras áreas, lo que indica que esta área está constituida por un solo estrato. Una explicación es que al no desmontarse esta área los individuos se encuentran en una fuerte competencia por la captación solar, formándose una comunidad vegetal con una sola cohorte. Mientras, en las otras áreas se eliminaron en su totalidad los individuos vegetales y en la actualidad se encuentran individuos formando copas anchas y la altura está relacionada a la velocidad potencial de crecimiento.

\section{CONCLUSIONES}

Con la presente investigación se concluye que áreas con diferente historial de uso silvoagropecuario muestran uniformidad de dimensiones diamétricas y de altura, con una cohorte dominante (estrato III). La riqueza específica del total de las áreas evaluadas fue de 27 especies, donde 24 se presentaron en el área de ganadería, 20 en matarrasa y 19 en agricultura, compartiendo 14 especies. De acuerdo a la diversidad de especies, el área matarrasa no mostró diferencias significativas con agricultura y ganadería, mientras que agricultura y ganadería sí presentaron diferencia, teniendo mayor diversidad el área de agricultura. Pese a que el área de ganadería tiene una mayor riqueza específica presentó menor diversidad, ya que el área se encuentra constituida por escasas especies con alta abundancia y muchas especies con escasa abundancia.

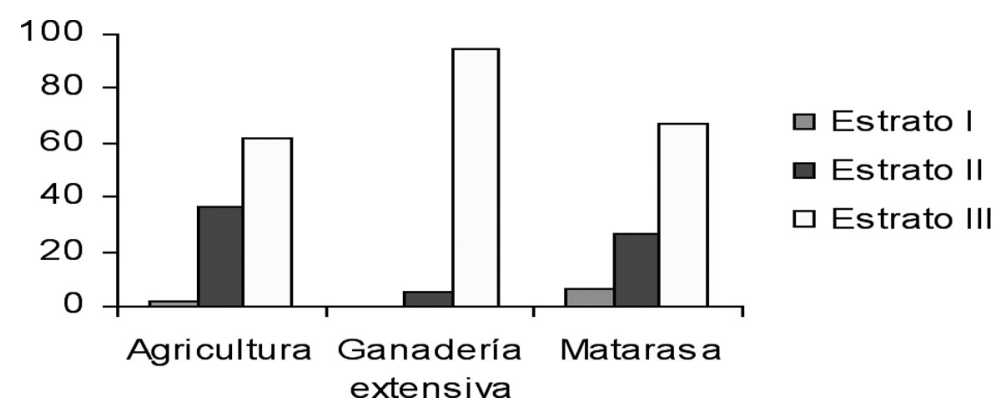

Figura 4. Distribución de la abundancia porcentual de los valores de la distribución vertical de las especies de las áreas evaluadas. 


\section{RECONOCIMIENTOS}

A la Facultad de Ciencias Forestales de la Universidad Autónoma de Nuevo León por las facilidades otorgadas en la realización de esta investigación.

\section{REFERENCIAS}

Aguirre, O., H. Kramer y J. Jiménez. 1998. Strukturuntersuchungen in einem Kifern-Durchforschungsversucht Nordmexikos. Allg Forest-u J Ztg. 169: 213-219.

Aguirre, O., J. Jiménez, H. Kramer y A. Akça. 2003a. Análisis estructural de ecosistemas forestales en el Cerro del Potosí, Nuevo León, México. Ciencia UANL 6(2): 219-225.

Aguirre, O., G. Hui, K. Gadow y J. Jiménez. 2003b. An analysis of spatial forest structure using neighbourhood-based variables. Forest Ecology and Management 183: 137145.

Alanís, E. 2006. Diversidad de especies arbóreas y arbustivas en áreas con distinto historial de uso antropogénico en el matorral espinoso tamaulipeco. Tesis de Maestría, Facultad de Ciencias Forestales, Universidad Autónoma de Nuevo León, Linares, N.L., México.

Alanís, E., J. Jiménez, O. Aguirre, E. Treviño, E. Jurado y M. González. 2008a. Efecto del uso del suelo en la fitodiversidad del matorral espinoso tamaulipeco. Revista Ciencia UANL 11(1): 56-62.

Alanís, E., J. Jiménez, D. Espinoza, E. Jurado, O. Aguirre y M. González. 2008b. Evaluación del estrato arbóreo en un área restaurada postincendio en el Parque Ecológico
Chipinque. Revista Chapingo Serie Ciencias Forestales y del Ambiente 14(2): 13-118.

Camargo, S. y V. García. 2001. El género Mimosa L. (Fabaceae) y la restauración ecológica. Contactos (UAMI) 39: 34-42.

Castellanos, F., E. Treviño, O. Aguirre, J. Jiménez, M. Musalem y R. López. 2008. Estructura de bosques de pino pátula bajo manejo en Ixtlán de Juárez, Oaxaca, México. Madera y Bosques 14(2): 51-63.

Corral, J., O. Aguirre, J. Jiménez y S. Corral. 2005. Un análisis del efecto del aprovechamiento forestal sobre la diversidad estructural en el Bosque Mesófilo de Montaña "El Cielo", Tamaulipas, México. Investigaciones Agrarias Sistema de Recursos Forestales 14(2): 217-228.

Del Río, M., F. Montes, I. Cañellas y G. Montero. 2003. Índices de diversidad estructural en masas forestales. Investigaciones Agrarias Sistema de Recursos Forestales 12(1): 159-176.

Diamond, D., D. Riskind y S. Orzell. 1987. A framework for plant community classification and conservation in Texas. Texas Journal of Science 39: 202-221.

Durán, E., J. Meave, E. Lott y G. Segura. 2006. Structure and tree diversity patterns at the landscape level in a mexican tropical deciduous forest. Boletín de la Sociedad Botánica de México 79: 43-60.

Edwards, P., R. May y N. Webb. 1993. Large-scale ecology and conservation biology. Gran Bretaña. 1991 p.

Espinoza, R. y J. Návar. 2005. Producción de biomasa, diversidad $\mathrm{y}$ 
ecología de especies en un gradiente de productividad en el matorral espinoso tamaulipeco del nordeste de México. Revista Chapingo Serie de Ciencias Forestales y del Ambiente 11(1): 25-31.

Estrada, E., C. Yen, A. Delgado y J. Villarreal. 2004. Leguminosas del centro del estado de Nuevo León, México. Anales del Instituto de Biología, UNAM. Serie Botánica 75(1): 78-85.

Flores, E., J. Frías, P. Jurado, J. Figueroa, V. Olalde y A. Valdivia. 2006. Influencia del gatuño (Mimosa monancistra Benth) en la infiltración de agua y la cantidad de forraje en pastizales con diferente grado de disturbio en el altiplano central mexicano. Técnica Pecuaria de México 33(1): 27-40.

Foroughbakhch, R. y R. Peñaloza. 1988. Introducción de 10 especies forestales en el matorral del noreste de México. Reporte Científico No. 8, Facultad de Ciencias Forestales, UANL. 33 p.

Franco, J., J. De la Cruz, G. Cruz, R. Rocha, S. Navarrete, M. Flores, M. Kata, C. Sánchez, A. Abarca y S. Bedina. 1989. Manual de ecología. Trillas, México. 94 p.

Füldner, K. 1995. Zur strukturbeschreibung in mischbeständen. Forstarchiv 66: 149-161.

Füldner, K. y K. Gadow. 1994. How to define a thinning in a mixed deciduous beech forest. In, Mixed Stands. Proceeding from the Symposium of the IUFRO Working Group S4.01-03 and S4.01-04. Lousa \& Coimbra, Portugal. pp 31-42.

Gadow, K. 1993. Zur Bestandesbesschreibung in der Forsteinrichtung. Forst und Lodz. 21: 601-606.
Gadow, K., G. Hui y M. Albert. 1998 Das Winkelmass - ein Strukturparameter zur Beschreibung der Individualverteilung in Waldbeständen. Centralblatt für das gesamte Forstwesen 115(1): 1-9.

García, J. y E. Jurado. 2008. Caracterización del matorral con condiciones prístinas en Linares N.L., México. Ra Ximhai 4(1): 1-21.

Gómez, A. 2000. Evaluación de áreas forestales de matorral utilizando un inventario multifásico. Tesis de Maestría, Facultad de Ciencias Forestales, Universidad Autónoma de Nuevo León, Linares, NL, México. 62 p.

González, G., O. Aguirre y G. Rodríguez. 2006. Caracterización estructural del estrato arbóreo en un bosque tropical subcaducifolio de la costa de Jalisco. Avances en la investigación científica en el CUCBA. pp 85-91.

González, H. e I. Cantú. 2001. Adaptación a la sequía de plantas arbustivas de matorral espinoso tamaulipeco. Revista Ciencia UANL4(4): 454461.

González, M. 1985. El límite sur de la provincia biótica tamaulipeca. II Simposio Internacional sobre la provincia biótica tamaulipeca, UAT y UNAM. Resúmenes.

González, M., E. Treviño y E. Jurado. 1997. Diversidad florística de la vegetación secundaria en un área de matorral del noreste de México. Journal International of Phytology 83(4): 280-281.

Graz, P. 2004. The behaviour of the species mingling index Msp in relation to species dominance and 
dispersal. European Journal of Forest Research 1: 87-92.

Grela, I. 2003. Avaliação do estádio sucessional de uma floresta subtropical de "quebrada" na região norte do Uruguay. Acta Bot. Bras. 17(2): 315-324.

Heiseke, D. y R. Foroughbakhch. 1985. El matorral como recurso forestal evaluación de 2 tipos de matorral en la región de Linares, N. L. Reporte Científico No. 1, Facultad de Ciencias Forestales, UANL. 33 p.

Huang, W., V. Pohjoenen, S. Johansson, M. Nashanda, L. Katigua y O. Luukkanen. 2003. Species diversity, forest structure and species composition in Tanzania tropical forests. Forest Ecology Management 173: 11-24.

Jiménez, J., O. Aguirre y H. Kramer. 1998. Bestandesstrukturanalyse in ungleichaltrigen Kiefern Wacholder Eichen Mischwald Nordostenmexikos. Forstachiv 69(6): 227-234.

Jiménez, J., O. Aguirre, H. Kramer y A. Akça. 2001a. Análisis y monitoreo en ecosistemas multicohortales. Programa de manejo y conservación en recursos naturales. Retos y Posibilidades de Cooperación Científica a Nivel Regional y Suprarregional para un desarrollo Sustentable. Seminario DAAD. Costa Rica.

Jiménez, J., O. Aguirre y $\mathrm{H}$. Kramer. 2001b. Análisis de la estructura horizontal y vertical en un ecosistema multicohortal de pino-encino en el norte de México. Investigaciones Agrarias Sistema de Recursos Forestales 10(2): 355-366.

Jurado, E. y N. Reid. 1989. Influencia de factores edáficos, topográficos $y$ perturbaciones sobre el matorral espinoso tamaulipeco en Linares N.L. Reporte Científico No. 10, Facultad de Ciencias Forestales, UANL. $p$ 4-5, 17-18.

Köhler, A. 1951. Vorratsermittlung in Buchenbeständen nach Stammzahldurchmesser und Stammabstand. Allgemeine Forst $u$. Jagdzeitung 123: 69-74.

Kramer, H., J. Jiménez y O. Aguirre. 1999. Zur Durchmesser - und Altersdifferenzierung in ungleichaltrigen NadelLaubholz-Mischwald. Forstarchiv. 70: 138-142.

Magurran, A. 2004. Measuring biological diversity. Blackwell. pp 106-121.

Mani, S. y A. Parthasarathy. 2006. Tree diversity and stand structure in inland and coastal tropical dry evergreen forest of peninsular India. Current Science 90(9): 1238-1246.

Mason, W., T. Connolly, A. Pommerening y C. Edwards. 2007. Spatial structure of semi-natural and plantation stands of scots pine (Pinus sylvestris L.) in northern Scotland. Forestry 80(5): 567-586.

Montes, F., I. Cañellas, M. Del Río y G. Montero. 2004. The effects of silviculture on the structure in mature Scots pine stands. In, F. Anderson, Y. Birot y R. Paivinen (Eds.) Towards the sustainable use of Europe's forest. Forest ecosystem and landscape research scientific challenges and opportunities. EFI Proceedins $n^{\circ}$ 49. pp. 61-71.

Mueller-Dombois, D. y H. Ellenberg. 1974. Aims and methods of vegetation ecology. John Wiley. 547 p. 
Pommerening, A. 1997. Eine Analyse neuer Ansätze zur Bestandesinventur in Strukturreichen Wäldern. Dissertation, Forstl. Fakultät d. Universität Göttingen, Cuvillier Verlag, Göttingen. 150 p.

Pretzsch, H. 1996. Strukturvielfalt als Ergebnis waldbaulichen Handels. Deutscher Verband Forestlicher Forschungsanstalten. Sekt. Ertragskunde. Jahrestagung Nehresheim. pp 134-154.

Ramírez, R., H. González, R. Ramírez, M. Cerrillo y A. Juárez. 2006. Seasonal trends of macro and micro in 10 browse species that grow in northeastern Mexico. Animal Feed Science and Technology 128: 155-164.

Ruiz, L. 2005. Caracterización estructural del matorral espinoso tamaulipeco. Tesis de Maestría, Facultad de Ciencias Forestales, Universidad Autónoma de Nuevo León, Linares, NL, México. pp 87-93.

Rzedowski, J. 1981. Vegetación de México. Limusa, México. 432 p.

Segura, G., P. Balvanera, E. Durán y A. Pérez. 2003. Tree community struc- ture and stem mortality along a water availability gradient in a mexican tropical dry forest. Plant Ecology 169(2): 259-271.

Shannon, C. 1948. The mathematical theory of communication. The Bell System Technical Journal 27: 379423

Solís, R., O. Aguirre, E. Treviño, J. Jiménez, E. Jurado y J. Corral. 2006. Efecto de dos tratamientos silvícolas en la estructura de ecosistemas forestales en Durango, México. Madera y Bosques 12(2): 49-64.

Van, M., M. Martinez y F. Bongers. 2006. Community dynamics during early secondary succession in a mexican tropical rain forests. Journal of Tropical Ecology 22(6): 663-674.

Vorãák, J., J. Merganic y M. Saniga. 2006. Structure diversity change and regeneration processes of the norway spruce natural forest in Babia-Hora NNR in relation to altitude. Journal of Forest Science 52(9): 399-409.

Zar, J. 1999. Biostatistical analysis. 4ta. ed. Prentice-Hall. 663 p. y M. González-Tagle. 2009. Análisis sobre el efecto del uso del suelo en la diversidad estructural del matorral espinoso tamaulipeco. Madera y Bosques 15(3): 5-20. 